\section{Anämie korrigieren bringt nix}

Die Korrektur einer bestehenden Anämie hat bei Patienten mit Herzinsuffizienz in einer großen Studie (RED-HF) nicht zu der erhofften Reduktion von Mortalität und Morbidität geführt. Anämie sei bei Herzschwäche nur ein Marker für eine schlechte Prognose, aber kein therapeutischer Ansatzpunkt, folgern die Studienautoren aus dem enttäuschenden Ergebnis. An der Studie waren 2278 Patienten mit systolischer Herzinsuffizienz und Anämie ( $\mathrm{Hb}$-Werte zwischen $9 \mathrm{~g} / \mathrm{dl}$ und $12 \mathrm{~g} / \mathrm{dl}$ ) beteiligt. Zielvorgabe für die $\mathrm{Hb}$ Anhebung war ein Wert von mindestens $13 \mathrm{~g} / \mathrm{dl}$ und maximal $14,5 \mathrm{~g} / \mathrm{dl}$.

N Engl J Med 2013, 368:1210

\section{Daten und Fakten}

\section{4}

betrug im Jahr 2010 bei der Herzinsuffizienz die Zahl der vollstationär behandelten Fälle pro 100.000 Einwohner (stationäre Morbiditätsziffer) in Deutschland. Diese Zahl nennt der "deutsche Herzbericht 2011“. Im Jahr 1955 lag sie noch bei 275. Innerhalb von 15 Jahren war demnach ein deutlicher Anstieg zu verzeichnen. Die Sterbeziffer (Todesfälle pro 100.000 Einwohner) bei Herzinsuffizienz ist dagegen zwischen 2000 und 2010 von 69,3 auf 59,1 gesunken.

\section{Infos im Internet}

Mehr Beiträge zum Thema finden Sie in unserem Dossier Herzinsuffizienz, unter www. springermedizin.de. Sie finden die Beiträge, indem Sie 215376 in die Suche eingeben.

Die neuen europäischen Leitlinien zur Herzinsuffizienz sind auf der ESC-Website www.escardio.org zu finden.

Aktuelle Informationen zu kardiologischen Themen bietet das Onlineportal www.kardiologie.org, das von der DGK mit Springer Medizin als Partner betrieben wird.

ACE-Hemmer \& Co. bei diastolischer Herzschwäche

\title{
Gesamtsterberate bleibt gleich
}

Bei etwa jedem zweiten Patienten mit Herzinsuffizienz ist die systolische linksventrikuläre Funktion noch weitgehend normal.

Das Wissen über die Herzinsuffizienz mit erhaltener Auswurffraktion (diastolische Herzinsuffizienz) ist noch immer relativ spärlich. Diese Form der Herzschwäche wird häufig bei älteren Patienten, bei Frauen sowie in Assoziation mit Bluthochdruck und Linksherzhypertrophie beobachtet. Da es nach wie vor an Therapien mit nachgewiesenem Nutzen mangelt, enthalten auch die aktualisierten Leitlinien keine neuen Therapieempfehlungen. In großen Studien hat sich gezeigt, dass Pharmakotherapien wie ACE-Hemmer oder Angiotensin-Rezeptorblocker, die bei systolischer Herzinsuffizienz nachweislich eine Reduktion von Morbidität und Mortalität bewirken, einen vergleichbaren Nutzen bei diastolischer Herzinsuffizienz vermissen lassen. Um sich ein Bild von der Wirksamkeit bisher untersuchter Pharmakotherapien $\mathrm{zu}$ machen, hat eine australische Arbeitsgruppe die Daten von 30 Studien mit insgesamt fast 54.000 Patienten ausgewertet. Die Ergebnisse zeugen von sehr bescheidenen Therapieerfolgen.

\section{Verlängerte Belastungsdauer}

Für keine untersuchte einzelne Wirkstoffklasse wie ACE-Hemmer, Angiotensin-Rezeptorblocker, Betablocker sowie Vasodilatatoren oder Kombinationen konnte im Beobachtungszeitraum von knapp 19 Monaten ein günstiger Einfluss auf die Gesamtsterberate nachgewiesen werden. Ebenso wenig gab es in den Studien Anzeichen für eine Verbesserung der diastolischen linksventrikulären Funktion durch die geprüften Therapien.

Einzig greifbarer Erfolg: In sechs kontrollierten Studien, in denen die Wirkung von kombinierten Therapien auf die Belastungskapazität der Patienten im Blickpunkt stand, wurde eine signifikante Verlängerung der Belastungsdauer bei Therapie mit Vasodilatatoren oder chronotropen Substanzen wie Verapamil oder Betablocker beobachtet.

(ob)

Ärzte Zeitung, 03.07.2013

\section{Verbesserte Parameter}

\section{Teilerfolg für Spironolacton}

Die Suche nach Therapiemöglichkeiten für Patienten mit diastolischer Herzinsuffizienz geht weiter.

Mithilfe von 422 Patienten mit diastolischer Herzinsuffizienz und moderater Symptomatik wurde in der Aldo-DHFStudie Spironolacton auf seine klinische Wirksamkeit bei dieser Indikation geprüft. Am Ende der einjährigen Beobachtungszeit zeigten sich die dopplerechokardiografischen Parameter der diastolischen linksventrikulären Funktion signifikant verbessert, im Vergleich zu Placebo. Beobachtet wurde auch eine Abnahme des kardialen Biomarkers NT-proBNP, als Ausdruck einer verbesserten neuroendokrinen Aktivität sowie eine Abnahme des linksventrikulären Massenindexes ( $\mathrm{Re}$ verse Remodeling). Die körperliche Leistungsfähigkeit wurde hingegen nicht verbessert. Die Gehstrecke beim 6-MinutenGehtest nahm sogar leicht ab. Auch die Lebensqualität blieb unverändert. Die funktionellen und strukturellen Verbesserungen waren größtenteils unabhängig von der Blutdrucksenkung durch Spironolacton, so die Autoren.

(ob)

JAMA 2013, 309:781 